\title{
How can we improve adherence to therapy by patients with rheumatoid arthritis?
}

\author{
Bart JF van den Bemt and Wim GJM van Lankveld
}

Disease modifying antirheumatic drugs (DMARDs) minimize disease activity, reduce radiological progression and improve long-term functional outcomes in patients with rheumatoid arthritis (RA); however, as with other chronic conditions, adherence rates to prescribed medicine regimes in this patient group are low, varying from 30-80\%. Improving adherence to therapy could dramatically improve the efficacy of medical treatments and reduce costs, but identifying factors associated with nonadherence and applying interventions in an effective manner can be difficult.

Knowledge of factors associated with medication adherence in RA could help physicians to identify patients who would benefit from intervention in this regard, and could enable these approaches to be tailored to combat the individual's primary reasons for nonadherence. Few studies, however, have examined adherence to DMARDs in patients with RA. A wide range of variables have been identified as being linked to adherence to medication in general, but none of these variables have been consistently shown to be related to adherence across the different studies. Neither, sociodemographic, nor biomedical, nor psychological variables, therefore, seem to be strong and consistent predictors of adherence to therapy (Vermeire E et al. [2001] J Clin Pharm Ther 26: 331-342; Donovan JL [1995] Int J Technol Assess Health Care 11: 443-455). In addition, most interventions to improve adherence to therapy have proven to be of limited effectiveness.

Adherence is a complex phenomenon that cannot be explained by a single factor or psychological variable. Several models have, therefore, been developed in an attempt to understand nonadherence. The most widely used model, the Health Beliefs Model, hypothesizes that individuals will adhere to health regimens if they regard themselves as being susceptible to the condition in question, if the condition has serious consequences, if the action would be beneficial, and if they feel that

....besides
tackling
practical
barriers ...
clinicians
should be
sensitive to
a patient's
personal
beliefs that
might affect
medication
adherence...

BJF van den Bemt is an Outpatient Pharmacist and a Clinical Scientist in the Department of Pharmacy and WGJM van Lankveld is a Psychologist and a Senior Researcher in the Department of Rheumatology, both at the Sint Maartenskliniek, St Maartens Hospital, Nijmegen,

The Netherlands.

Competing interests

The authors declared no

competing interests.

www.nature.com/clinicalpractice doi:10.1038/ncprheum0672 barriers to action are outweighed by the benefits. Patients consider whether their beliefs about the necessity of medication outweigh their concerns about potential adverse effects of taking them. The patient's beliefs about the necessity of medication and the concerns about medication can be assessed using the Beliefs about Medicines Questionnaire (BMQ; Horne R [1999] J Psychosom Res 47: 491-495). The BMQ has been validated for use in patients with chronic illnesses and has been shown to predict adherence to treatment amongst other groups of patients with chronic conditions (e.g. people with asthma).

Most people with RA report positive beliefs about the necessity of their medication. Levels of concern are, nonetheless, also high and seem to be related to nonadherence. These data were confirmed in our cross-sectional study of 228 people with RA. In this study, only $68 \%$ of the patients adhered to their prescribed drugs as assessed with the validated Compliance Questionnaire Rheumatology (CQR; de Klerk E et al. [2003] J Rheumatol 30: 2469-2475). We found that $52 \%$ of nonadherent patients had overall positive beliefs about the necessity of their drug; however, $91 \%$ of the nonadherent patients also had one or more concern about potential adverse effects, particularly over the long term. This indicates that interventions to optimize therapy adherence should concentrate on patient concerns rather than on the necessity of medication.

In conclusion, nonadherence leads to decreased efficacy of medical treatment. Many factors seem to influence adherence, and, therefore, interventions to improve adherence should be tailored to individual's primary reason(s) for not taking their medication. Besides tackling practical barriers, such as forgetfulness, clinicians should be sensitive to a patient's personal beliefs that might affect medication adherence, and should discuss with their patients any concerns that they raise about prescribed medications. 